\title{
COMPETENCIA - ENTRE SIGNIFICADO Y CONCEPTO
}

Paula Batista
Amândio Graça
Zélia Matos
Universidad de Oporto
"words or expressions that may always have the same meaning
when applied to one kind of thing, but have different meaning
when applied to another kind of thing"

(Flew, 1979: 11)

\section{RESUMEN}

Este trabajo tiene como principal propósito contribuir a la clarificación del concepto de competencia, a la luz de los desafíos que actualmente tienen la formación y el desarrollo de la competencia. Se comienza por reflexionar sobre las cuestiones léxicas, relacionadas con los significado(s) de competencia, luego nos centramos en el debate en torno al concepto(s) de competencia, preferentemente en áreas de formación, evaluación y desarroIlo profesional. Tras el análisis efectuado emerge la idea de que no existe un solo constructo teórico de "competencia", sino que coexisten varios en campos conceptuales diferenciados ya que el concepto de competencia es un concepto complejo, holístico, integrador y de naturaleza relacional. La competencia se presenta como algo situacional; ocurre en la acción, no es directamente observable; se relaciona con el éxito, pero no se confunde con el desempeño concreto.

\section{ABSTRACT}

The main purpose of this study is to contribute to the clarification of the concept of competence in light of the new challenges the education and development of competence has to face nowadays. After a brief review of lexical issues related to the meaning(s) of competence, the analysis is focused on the debate concerning the concept of competence occurring within such areas as professional education, evaluation, and development. The analysis support the idea that rather than a unique construct of competence, there are several dissimilar conceptual fields, portraying competence as a complex, holistic, integrative, and relational concept. Competence is situational, it is reflected in action, it is not directly observed, it is related to the outcomes but it should never be confused with any particular performance. 


\section{Introducción}

Este trabajo tiene como principal propósito contribuir a la clarificación del concepto de competencia, colocando en "confronto" la gran diversidad de ideas, muchas veces contradictorias, que se entrecruzan en esta área de investigación. Es también nuestra intención ir más allá del "inventario" de las diferentes nociones y usos de la competencia, procurando identificar las posibilidades y viabilidad del uso del concepto de competencia.

Cuando lanzamos una mirada sobre las cuestiones de la competencia, fácilmente constatamos que el término competencia se utiliza de forma muy poco cuidada y muchas veces indiscriminadamente. La generalidad de los estudios se basa en "arenas movedizas" y recorre perspectivas muy atomistas o muy generalizadas acabando por revelarse de poca utilidad. En este contexto poco claro, consideramos fundamental esclarecer el cuadro teórico en que nos movemos, de forma que la investigación empírica en el área se efectúe de forma sustentada y contextualizada. Otro aspecto importante que nos movió fueron los nuevos desafíos que la sociedad coloca en los más diversos sectores, sobre todo a nivel de la enseñanza superior, más específicamente en los programas de formación inicial. La necesidad de reformulación de los programas de formación inicial, conduce a la necesidad de proceder a una (re)interpretación del concepto de competencia, bajo nuevas realidades, para que la reformulación, ya en curso en muchas áreas de formación, se revele fructífera. En nuestra opinión los trabajos de clarificación y (re)colocación del concepto de competencia deben ser el punto de partida para las cuestiones investigadas después, sobre todo las relacionadas con la formación, percepción y desarrollo de la competencia, o bien la investigación podrá revelarse extremadamente improductiva por la ausencia de consistencia de las conceptualizaciones e inadecuaciones frente a las exigencias dictadas por los nuevos paradigmas en emergencia. Utilicemos de nuevo el ejemplo de la enseñanza superior, cuyas reformas legislativas nos muestran el sentido de un pasaje del paradigma centrado en el conocimiento hacia un paradigma centrado en las competencias.

Tomando como punto de referencia la naturaleza conceptual del trabajo y su propósito central, estructuramos el trabajo en tres grandes etapas. En la primera etapa buscamos clarificar los referenciales básicos utilizados cuando se habla de competencia, recurriendo al campo de la lingüística para intentar situar el significado de "palabra" y "término". Analizamos e interpretamos la existencia, o no, de diferencias entre "significado" y "concepto". Nos propusimos, además, hacer una exploración léxica del significado de competencia, partiendo de su raíz etimológica y elaboramos una síntesis de las diferentes designaciones atribuidas a competencia, en un diversificado conjunto de idiomas, e incluso presentamos la diversidad de significados y palabras que usualmente se utilizan como sinónimos de competencia. En la segunda etapa nos centramos en la exploración terminológica del concepto de competencia; también aquí buscamos llegar a un esquema representativo de las principales ideas agregadas al concepto de competencia. En la tercera y última etapa, realizamos una síntesis conclusiva de la revisión de la literatura efectuada a la que añadimos una propuesta conceptual de encuadramiento del concepto de competencia. Enseñamos aún posibles caminos a seguir para profundizar la investigación en esta área, enfocando, de manera especial, los desafíos que actualmente se colocan a la enseñanza superior, por el paso del paradigma del conocimiento hacia el paradigma de las competencias, lo que en consecuencia obliga a una reformulación de los currículos y los programas de formación inicial. 
Hecho este encuadramiento general pasamos ahora a tratar el tema en si mismo, intentando en primer lugar clarificar los referenciales básicos que deben preceder la exploración de cualquier temática sobre competencia.

La exploración de la temática de la competencia nos depara, desde luego, un escenario de confusión. Según Carr (1993), el centro de esta confusión está formado por un conjunto de equívocos que resultan, en parte, porque la palabra competencia se aplica en contextos muy diversificados y con diferentes significados. Una forma de intentar disipar esta confusión pasa por la clarificación del referencial utilizado cuando se "discurre" sobre la competencia. En este sentido es pertinente esclarecer si el referencial utilizado es el significado (ligado a la palabra) o el concepto (ligado al término) ${ }^{11}$. Lara (2004) ilustra bien esta diferencia al enunciar que "el término es un signo lingüístico que difiere de la palabra; mientras que la palabra tiene propiedades pero tiene muchos significados, un término, al contrario, es una palabra contextualizada en el discurso, teniendo, en consecuencia, un referente de interpretación." Esta primera diferenciación entre término y palabra nos conduce a una segunda distinción, la diferencia entre significado y concepto.

El significado se relaciona con las cuestiones lingüísticas y está directamente ligado a la palabra. El concepto es terminológico, se relaciona con el término y se refiere a un ámbito específico.

Teniendo como base estos criterios, consideramos importante iniciar el proceso de descubrimiento de la "competencia" a través de la exploración léxica, en busca de los diferentes significados de acuerdo con el uso que se hace de la palabra, para que, a continuación, nos centremos en el concepto de competencia, que exige una búsqueda de definición terminológica (que demuestra el concepto dentro de un área específica).

\section{Significado(s) de la palabra competencia}

En la medida en que asumimos la distinción entre el significado y el concepto, cuando analizamos el significado no tenemos como objetivo presentar una definición terminológica de "competencia", sino buscamos identificar el uso que se hace de la palabra "competencia". El lugar de búsqueda más común, a pesar de la superficialidad que conlleva, pero sin dejar que se revista de algún interés, es la raíz etimológica de competencia. La etimología nos revela que la palabra "competencia" proviene del latín tardío: competencia - que significa proporção (Diccionario de la Lengua Portuguesa, Porto Editora); proportion, rapport exact (Dictionnaire de I'Académie, 9ème éd.); agreement (The ORB: On line referente book for medieval studies), y que a su vez deriva del verbo latín competere ${ }^{[2]}$, en el que "com" (cum) significa juntos y "petere" significa procurar, buscar, converger. De competere deriva también el verbo competir, así como el adjetivo competente (competens), y el nombre competición (competitio).

[1] En este trabajo asumimos que existe una diferencia entre término y palabra, esto a pesar de que algunas corrientes contemporáneas relativicen esa distinción (Gaudin, 1995; Cabré, 2000).

[2] "encontrar-se no mesmo sítio; coincidir" (Dicionário da Língua Portuguesa, Porto Editora). 
Además de la raíz etimológica, el diccionario nos indica un conjunto de significados que se atribuyen a competencia, así como definiciones diferenciadas de acuerdo con el dominio de aplicación.

A partir de la traducción de la palabra inglesa competence para diversas lenguas (Cuadro 1), podemos constatar la presencia de palabras con la misma raíz de competencia, que provienen directamente del latín, o por vía indirecta, de otras lenguas.

Cuadro 1. Traducción de competence a partir del inglés -> MultiLingual Online Dictionary - a Bilingual Dictionary from ECTACO.

\begin{tabular}{|c|c|}
\hline Bulgarian & 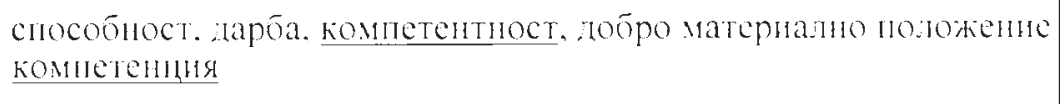 \\
\hline Czech & pravomoc, kompetentnost, kompetence \\
\hline German & $\begin{array}{l}\text { fähigkeit, kompetenz, sicherheit, tüchtigkeit, zuständigkeit, } \\
\text { zuständigkeitsbereich, einkommen }\end{array}$ \\
\hline Spanish & $\begin{array}{l}\text { competencia, capacidad, habilidad, posibilidad, oportunidad, } \\
\text { aptitud, capacidad física }\end{array}$ \\
\hline Estonian & $\begin{array}{l}\text { kompetents, võimkond, võimed, võimupiirid, õigusvõime, õiguspä- } \\
\text { devus, oskus: oskused, kompetentsus, pädevus, asjatundlikkus }\end{array}$ \\
\hline French & compétence, capacité \\
\hline Italian & competenza, capacità \\
\hline Latvian & spējas, dotības, turt̄ba, kompetence \\
\hline Dutch & $\begin{array}{l}\text { competentie, bevoegdheid, bekwaamheid, rechtsbevoegdheid, } \\
\text { welgesteldheid }\end{array}$ \\
\hline Polish & znajomość rzeczy, fachowość, dostatek, kompetencja \\
\hline Portuguese & $\begin{array}{l}\text { competência, suficiência, capacidade, habilidade, alcance, vida } \\
\text { confortável, alçada }\end{array}$ \\
\hline Romanian & $\begin{array}{l}\text { competență, pricepere, clarificare, abilitate, capacitate, resort, } \\
\text { atributie, destoinicie, îndestulare, viaţă îndestulaţă }\end{array}$ \\
\hline Russian & $\begin{array}{l}\text { умение. компетенция, способность, компетентность. достаток. } \\
\text { хоропее материальное поножение. правомочность }\end{array}$ \\
\hline Slovak & $\begin{array}{l}\text { kompetencia, schopnost', kvalifikácia, spôsobilost', právomoc, } \\
\text { príslušnost' }\end{array}$ \\
\hline Albanian & kompetencë, kamje, kamuri, fuqi ligjore \\
\hline Swedish & kompetens, behörighet, inkomst, välstånd, duglighet \\
\hline
\end{tabular}


Un breve análisis de la correspondencia de palabras entre la lengua inglesa y las demás lenguas revela que la traducción automática no puede utilizarse indiscriminadamente. Basta con fijarse en los resultados de la traducción al portugués para comprobar que los significados de competence y competencia no pueden sobreponerse. Hay significados de competence que no encajan en los significados de competencia y viceversa. El caso de la lengua española es, respecto a este asunto, bastante esclarecedor, ya que el diccionario presenta dos entradas distintas para competencia, una muy corriente, que no puede traducirse por competence, ligada a la idea de competir, disputar; y otra con sentido de capacidad, aptitud (ver Cuadro 2). En el ámbito del Derecho, un juez o tribunal se puede declarar incompetente para tratar un asunto dado y por ello no está confesando su incapacidad o falta de preparación, sino considerando que no entra el asunto en cuestión dentro de su dominio. El uso de competencia en esta acepción no se hace en la lengua inglesa. Por otro lado, la traducción de una palabra por otra de diferente raíz etimológica requiere mayor cuidado; por lo tanto, es poco fiable utilizarlas como sinónimos. Hay que prestar atención que la palabra asume, en la mayor parte de las lenguas, significados muy diversos en función de la aplicación que se hace de ella.

Cuadro 2. Sinónimos y significados de "competencia" en varias lenguas.

\section{Inglés (Dictionary.com Unabridged (v 1.1))}

Competence (n.) (competency; competencie)

1. the quality of being competent; adequacy; possession of required skill, knowledge, qualification, or capacity: He hired her because of her competence as an accountant.

2. sufficiency; a sufficient quantity.

3. an income sufficient to furnish the necessities and modest comforts of life.

4. Law. (of a witness, a party to a contract, etc.) legal capacity or qualification based on the meeting of certain minimum requirements of age, soundness of mind, citizenship, or the like.

5. Embryology. the sum total of possible developmental responses of any group of blastemic cells under varied external conditions.

6. Linguistics. the implicit, internalized knowledge of a language that a speaker possesses and that enables the speaker to produce and understand the language. Compare performance.

7. Immunology. immunocompetence.

8. Geology. the ability of a fluid medium, as a stream or the wind, to move and carry particulate matter, measured by the size or weight of the largest particle that can be transported.

Español (Real Academia Española Diccionario de la Lengua Española 22. a edición) competencia

Cf. competir 
1. f. Disputa o contienda entre dos o más personas sobre algo.

2. f. Oposición o rivalidad entre dos o más que aspiran a obtener la misma cosa.

3. f. Situación de empresas que rivalizan en un mercado ofreciendo o demandando un mismo producto o servicio.

4. f. Persona o grupo rival. Se ha pasado a la competencia.

5. f. Am. Competición deportiva.

Cf. competente

1. f. incumbencia.

2. f. Pericia, aptitud, idoneidad para hacer algo o intervenir en un asunto determinado.

3. f. Atribución legítima a un juez u otra autoridad para el conocimiento o resolución de un asunto.

Francés (Dictionnaire de l'Académie, 9ème édition)

compétence (n.f.)

1. DROIT. Aptitude d'une autorité publique à accomplir des actes dans des conditions déterminées. La compétence du maire, du préfet. Cela n'est pas de sa compétence. Une affaire de compétence nationale, qui relève exclusivement de la souveraineté de l'État. Spécialt. Droit qu'un tribunal, un juge a de connaître de telle ou telle matière, de telle ou telle cause. Cette affaire relève de la compétence de tel tribunal. Décliner la compétence d'un tribunal. Compétence administrative, relative aux litiges qui concernent le service public. Compétence d'attribution ou matérielle, établie selon la nature de l'infraction. Compétence personnelle, au regard de la personne du délinquant (mineur, militaire). Compétence territoriale, établie en fonction des circonstances de lieu.

2. Capacité, fondée sur un savoir ou une expérience, que l'on reconnaît à une personne. Je ne doute pas de ses compétences professionnelles, de sa compétence. Cela est hors de ma compétence, dépasse mes compétences. Manquer de compétence. Par méton. Fam. La personne compétente elle-même. C'est une compétence dans sa spécialité.

3. LINGUIST. Aptitude théorique de toute personne parlant une langue à produire et à comprendre un nombre indéfini de phrases.

\section{Italiano (De Mauro il dizionario della lingua italiana)}

\section{Competènza (s.f.)}

1. CO I'essere competente; capacità ed esperienza in un determinato campo, in una determinata attività: $c$. in materia, ha molta $c$. in matematica, è un avvocato di grande $\mathrm{c}$.

2. TS dir., burocr., potere di emanare atti giuridici: la c. del tribunale, del parlamento, del prefetto | ambito d'azione di un organo giurisdizionale o amministrativo: questa causa rientra sotto la c. del foro di Torino.

3. CO estens., pertinenza: la faccenda non è di mia c. | sfera d'azione: questo non rientra nella c. del nostro ufficio | spec. al pl., compito, funzione: queste 
sono le competenze del capo.

4. CO spec. al pl., quanto compete, compenso, onorario: le competenze del medico, del notaio.

5. TS ling., nella terminologia chomskyana: insieme delle conoscenze e delle regole grammaticali e linguistiche che permettono al parlante di comprendere (c. passiva) e formare (c. attiva) un numero infinito di nuove frasi; sapere linguistico | in glottodidattica, sociolinguistica, linguistica testuale: capacità di servirsi di una determinata parte o di un determinato aspetto della lingua: c. lessicale, sintattica, testuale.

6a. TS embriol., capacità di un'area embrionale di dare origine a un determinato organo.

6b. TS biol., capacità di un organismo o di un organo di svolgere le proprie funzioni.

7. TS med., integrità funzionale delle valvole cardiache.

8a. TS geol., capacità di una corrente di trasportare detriti rocciosi.

8b. TS geol., tendenza di una roccia a fratturarsi piuttosto che a deformarsi plasticamente in seguito a sollecitazioni meccaniche.

9. RE tosc., gara, competizione; mettersi, stare a c. con qcn., competere, gareggiare con lui | non c'è c., non c'è paragone.

10. BU persona molto competente.

Portugués (UOL Michaelis Moderno Dicionário da Lingua Portuguesa)

\section{Competência}

1 (lat competentia)

1. Capacidade legal, que um funcionário ou um tribunal tem, de apreciar ou julgar um pleito ou questão. 2 Faculdade para apreciar e resolver qualquer assunto. 3 Aptidão, idoneidade. 4 Presunção de igualdade. 5 Concorrência, confronto. 6 Conflito, luta, oposição. Antôn (acepções 1, 2 e 3): incompetência.

2 (ingl competence)

Para a teoria gerativa, capacidade que tem o falante nativo de uma língua para entender e produzir um número infinito de orações.

En medio de la diversidad de sentidos y significados de "competencia" en cada lengua y de lengua para lengua, podemos observar la presencia constante de un significado asociado a las nociones de capacidad, de posesión de los prerrequisitos necesarios. En una consulta al significado de "competence" en dos diccionarios, el Concise Oxford Dictionary ${ }^{[3]}$ y el Macquarie Concise Dictionary ${ }^{[4]}$, Hager y Gonczi (1996) se

[3] Concise Oxford Dictionary - Competence (competency) - "denotes the ability to do something or the ability for a task".

[4] Macquarie Concise Dictionary - Competence - "the quality of being competent", (Where competent means "properly qualified or capable"). 
verificó que ambos diccionarios tienen como primera acepción persona competente, o tiene capacidad (ability o capability) que permite la conclusión satisfactoria de una tarea.

Este núcleo de significado torna la palabra atractiva para usarse como concepto; sin embargo, la palabra competencia forma parte de una familia de palabras en la cual diversas ideas se entrecruzan de forma íntima asumiendo sentidos diferentes en función del /de los uso/s y contextos. Por ejemplo, Mansfield (2004) encontró cinco usos diferentes, lo que, en su opinión, constituye un factor de confusión:

Competent (adjetivo)- Persona capaz de desempeñar los requisitos de un empleo (unas veces en un sentido minimalista, otras veces en un sentido opuesto);

Competence (nombre)- estado de ser competente.

Competence/s (nombre)- las tareas que la persona desempeña.

Competency/ies (nombre)- las características personales que soportan el desarrollo eficaz o de nivel;

Competencels (nombre)- un elemento de la competencia (una competencia).

En conformidad, según el autor, el significado de competencia oscila entre resultados, tareas y características personales, remitiendo para una miscelánea contradictoria de modelos comportamentales, holísticos y psicosociales.

Parente (2003) apuntó como uno de los principales problemas de la falta de clarificación del concepto de competencia, el hecho de que éste se utilice como presunto término técnico de forma abusiva y poco esclarecedora, habiendo sólo una simple reproducción de viejos significados de sentido común. La autora sugiere que se sustituya el término competencia por el término competencias. La confusión generada por el uso descuidado que el sentido común hace de las palabras, se extiende también a la literatura de las áreas científicas. Por ejemplo, en la literatura americana, nos encontramos con el uso indiscriminado de diferentes palabras para indicar un mismo significado, como sucede con el uso de palabras como: competence, skills, performance, ability, capability. Entre nosotros esta situación también ocurre, ya por los diferentes usos que el sentido común hace de competencia, ya por la falta de consenso, a nivel de investigación, en torno del uso más adecuado de las palabras. Al igual que competencia son utilizadas, de forma muy poco cuidada y nada esclarecedora, palabras como: "desempeño, autoridad, capacitación, responsabilidad, calificación", entre otras. Las variadísimas tentativas de establecer una terminología coherente (Boak, 1991, Tate, 1995; Winterton, 1999; Wooddruffe, 1991) han tenido, hasta ahora, muy poco impacto. Por ejemplo Boak (1991) dice que el término competency en el contexto americano es complementario al término competence utilizado en el Reino Unido.

En resumen, podemos decir que el significado de competencia enfatiza las ideas de realización y de aptitud y que las definiciones encontradas tienen como núcleo a la persona competente, la posesión de determinados atributos que permiten resolver con éxito una determinada tarea. Otro aspecto importante a tener en cuenta es que dependiendo de los usos que se hacen de la palabra "competencia" se obtienen varios significados que surgen, cuando se refieren a un área específica, sobrepasando el dominio del significado, pasando a pertenecer al dominio del concepto (definición terminológica). Hay que apuntar que todavía existe un aspecto que sobresale 
apareciendo un elemento unificador: el hecho de que el uso de competencia surge íntimamente asociado a la actividad profesional. Por último, es importante también recordar que en la utilización del término competencia mediante el sentido común, existe una semejanza con otros conceptos, hecha de forma poco cuidada, hecho que alerta para la necesidad del aumento de atención en la utilización del término a nivel de investigación, tornándose imprescindible la identificación del área de significación.

La configuración del significado de competencia, representado en la figura 1, procura ilustrar las principales ideas a las que llegamos en esta exploración inicial del significado de "competencia".

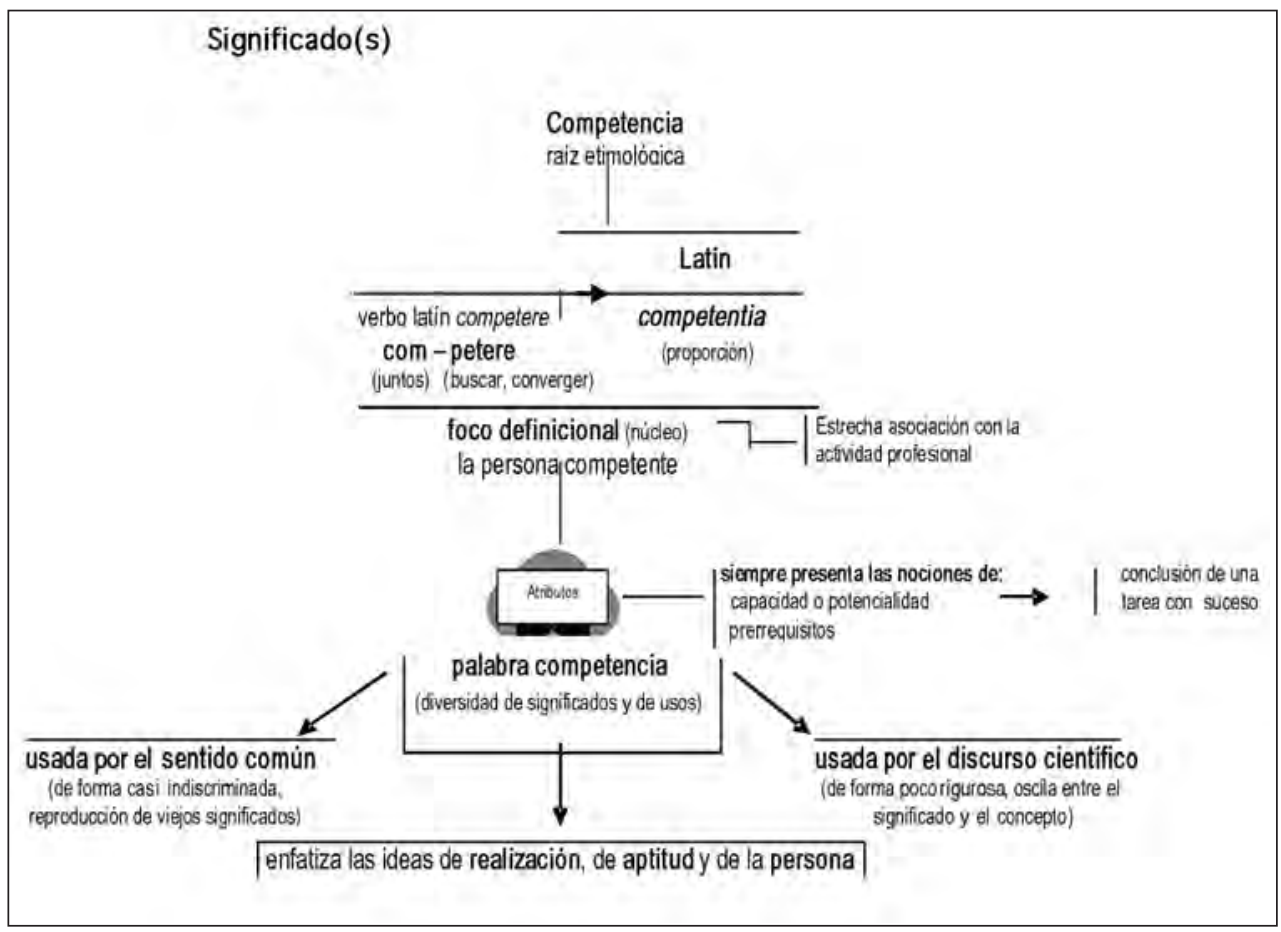

Figura 1. Síntesis de los aspectos relacionados con el significado de la palabra competencia.

Esta representación esquemática coloca en evidencia los principales aspectos que dan cuerpo al significado de competencia y que, resumiendo, podemos identificar de la siguiente manera: la palabra "competencia" tiene como foco a la persona, asume diferentes significados, se reporta a una actividad concreta realizada con éxito, surgiendo en estrecha asociación con la actividad profesional.

La frontera frágil que existe entre los dominios de significado y concepto torna aún más evidentes los problemas que existen en lo concerniente al estudio de la competencia. Pensamos que, cuando se haga investigación en esta área, sería necesario, desde el primer momento, situar el estudio en uno de los dominios: o en el dominio del significado o en el del concepto. Hay que tener en cuenta que los tra- 
bajos en el dominio del significado tienen una utilidad relativa, dado que no hace sentido buscar una definición consensual o seleccionar la mejor definición entre las disponibles. Consideramos que es posible considerar un significado que, aunque no es único y tiene un carácter histórico, puede sintetizarse de la siguiente forma: la competencia está directamente relacionada con el desempeño eficaz, puede observarse en acción y se asocia de forma estrecha con la persona.

Tomando como punto de partida estas líneas de fuerza procuraremos ahora sobrepasar el campo de definición del léxico para pasar al campo terminológico, es decir, al campo del concepto.

\section{Concepto(s) de competencia}

Siendo el término la expresión verbal de un concepto es de esperar que, de la semejanza del significado, nos surjan diferentes conceptos de competencia, cuya variabilidad resulta no sólo de la diversidad de campos de aplicación, sino también de campos conceptuales distintos dentro de un mismo campo de aplicación. En realidad, el concepto de competencia surge como un concepto poco pacífico, que se utiliza en diferentes contextos entendiéndose de distintas maneras.

El intento de conceptualizar la noción de competencia ha sido sistemático a lo largo de los tiempos (Carr, 1993; Hager, 1993; Hager \& Gonczi, 1996; Hoffman, 1999; Hyland, 1997; Le Deist \& Winterton, 2005; Short, 1984). Efectivamente, revisando la literatura acerca de esta temática, uno de los primeros aspectos que sobresale es la multiplicidad de definiciones que existen de competencia. Actualmente esta idea de falta de consenso todavía prevalece y está bien ilustrada por Le Deist y Winterton (2005) cuando dice que el concepto de competencia es un concepto borroso. Este autor considera que la confusión en torno al concepto es tal, que se torna imposible identificar o imputar una teoría coherente o llegar a una definición que incorpore y reconcilie las diferentes formas en que el término se utiliza.

Subrayando la dificultad de definir de manera precisa aquello a lo que verdaderamente la competencia se refiere, Hoffman (1999) apunta que el trazo de unión entre los diversos abordajes congrega el propósito de definir el desempeño competente, o las competencias, teniendo como horizonte la mejoría del desempeño profesional. Nótese, sin embargo, que tal y como en el ámbito de la lingüística generativa, hay que establecer una diferenciación entre competencia y desempeño (performance). Tal diferenciación permitirá evitar una definición estrecha de competencia (Noddings, 1984). En esta conformidad, se considera que la competencia es necesaria para un desempeño adecuado, pero las personas pueden ser igualmente competentes aunque presentan desempeños marcadamente distintos sin dejar de tener éxito. En el seguimiento de esta línea de argumentación Burgoyne (1988) defiende la idea de que "ser competente" es muy diferente de "tener competencias" pues ser competente implica cumplir con las exigencias impuestas por el trabajo y poseer las competencias no lleva necesariamente a un desempeño adecuado de las exigencias del trabajo. No basta entonces con tener condiciones para ser competente sino también es necesario saber movilizar esas condiciones en situaciones concretas. Por otro lado, cada desempeño particular depende de otros factores para bien de la competencia. Holmes (1992) ilustra la diferencia entre competencia y desempeño como ejemplo del conductor de coche: una persona puede ser un conductor competente, pero las condiciones atmosféricas, las condiciones del tráfico, el estado general 
(alcohol, fatiga) y la prisa del conductor pueden combinarse de tal forma que el desempeño de éste esté lejos del requerido por las normas de buena conducción e incluso infrinja las leyes más severas del código de carretera. Siendo así, el flojo desempeño no corresponde necesariamente a la falta de competencia. Como nos dice Boyatzis (1982, cit. In Holmes, 1992, p.4): "las acciones, sus resultados y las características necesarias que se movilizan/expresan no tienen necesariamente correspondencia una a una" ${ }^{\prime[5]}$.

Hager y Gonczi (1996) critican una visión reductora de competencia basada en el planteamiento de una serie de tareas discretas, en favor de un abordaje integrado que concibe la competencia en términos de conocimientos, capacidades, habilidades y actitudes evidenciadas en el contexto de un conjunto de tareas profesionales auténticas.

En una perspectiva integrada y holística, la competencia, al contrario de la perfomance (que puede ser directamente observable), sólo puede ser inferida a partir de múltiples desempeños. La competencia es relacional, dado que incorpora atributos (características personales relevantes como: conocimiento, habilidades y actitudes) y tareas, resultando esencialmente de relación entre capacidades y potencialidades de personas y la conclusión satisfactoria de una determinada tarea. Se rechaza así la reducción de la competencia en las tareas rutinarias, como su circunscripción a atributos personales genéricos, desligados de cualquier contexto de trabajo.

A pesar de que la generalidad de los autores incorpora la actitud como uno de los atributos que forman parte de los componentes de la competencia, otros presentan argumentos que colocan la actitud en un diferente nivel de los restantes atributos. Esta cuestión gana relevancia principalmente en el campo de formación y entrenamiento (training). Spencer y Spencer (1993) citan que la competencia puede ser comparable a un iceberg, pues tiene una parte visible y otra que no se ve, está sumergida. En el caso de la competencia el conocimiento y las habilidades tienden a ser visibles, luego, más susceptibles de enseñar, de desarrollar con el entrenamiento, mientras que las características de la persona como un autoconcepto, rasgos y motivos están escondidos, luego de difícil acceso y de más difícil desarrollo. En la continuidad de esta idea Parry (1996) distingue competencia "hard" de competencia "soft". La competencia "hard" se refiere a las habilidades específicas del trabajo (como conocimientos y habilidades relacionadas con el trabajo) y la competencia "soft" se refiere a la personalidad, rasgos, valores y estilos. Considerando estos aspectos tan distintos que componen las dos áreas de competencia, Parry considera que, en los procesos de formación y entrenamiento, la componente soft no debe incorporarse porque no puede desarrollarse con el entrenamiento. En este mismo sentido Matos (1989), basándose en autores como Ecke (1981) y Flach (1986), dice que la actitud no debe considerarse un elemento de la competencia, pero si un atributo determinante de ésta.

Otro aspecto que gana evidencia, en la exploración del concepto de competencia, es la constatación de que los atributos conocimientos y habilidades surgen incorporados en la generalidad de las nociones de competencia. A nivel de formación, sobre

[5] "actions, their results, and the necessary characteristics being expressed do not necessarily have a one-to-one correspondence". 
todo de la formación de profesores, el conocimiento, en sus varios dominios, es referenciado como la base de la competencia (eg. Flach, 1986; Shulman, 1987; Grossman, 1990). Todavía en esta línea de pensamiento, Perrenoud (1999) dice que la competencia asume un lugar central en el debate de formación dentro del contexto escuela. Competencia se asume como la capacidad de actuar eficazmente, en un determinado tipo de situación, apoyada en conocimientos, pero sin limitarse a ellos (Perrenoud, 1999:7). En la secuencia del concepto de competencia. Perrenoud (1999) defiende todavía conocimientos como: aspectos determinantes para identificar y resolver problemas, para preparar y formar decisiones, que, sin embargo, sólo tienen valor cuando están disponibles en el momento adecuado en sintonía con la situación (p. 53). A pesar de la relevancia que da al conocimiento, en el concepto de competencia, Perrenoud deja también bien clara la idea de que aunque el conocimiento es un elemento clave de la competencia, no es suficiente para ser competente.

En un análisis normativo del concepto de competencia, Pearson (1984) corrobora que el conocimiento, siendo necesario a la competencia, no es sinónimo de esta. De acuerdo con el autor, para que alguien sea competente en un área determinada, no basta con saber cómo hacer algo, pero, por otro lado, tampoco exige necesariamente que sea una autoridad en la materia. Una autoridad en determinada área/materia es alguien a quien las personas que laboran en esa área pueden recurrir cuando necesiten esclarecer una información técnica más sofisticada o profundizar un conocimiento, por lo que se admite que este tipo de información o conocimiento puede estar fuera del alcance de una persona que es competente en un determinado campo, pero no es una autoridad en el mismo.

Según Pearson (1984), la identificación de una persona competente conlleva decisiones a tres niveles:

Nivel de determinación del padrón deseable para una actuación satisfactoria.

Nivel de las habilidades que la persona debe poseer para poder actuar en ese registro.

Nivel de cómo evaluar si la persona en cuestión posee esos mismos requisitos.

Para el autor, el primero de estos niveles no es reducible a cuestiones de hecho, es también necesariamente de orden normativo, en la medida en que implica siempre un juicio de valor a cerca de lo que es deseable o apropiado. Pero, además de eso, el autor rechaza la pretensión behaviorista de poderse determinar anticipadamente y con exactitud las competencias básicas necesarias para actividades tan complejas y abiertas como la enseñanza. Rechaza que un "intelligent skill knowledge" (que requiere discernimiento, comprensión, sensatez, e inteligencia), se puede constituir a partir de un conjunto definido de "habitual skill knowledge" (habilidades específicas y rutinas aprendidas por entrenamiento). Considera por eso que la relación entre el concepto de competencia y la ideología behaviorista que subyace a muchos programas de formación es solo contingente.

Reforzando esta noción De Landsheere (1986) considera que la competencia no es una simple aplicación aislada de capacidades cognitivas, afectivas o psicomotoras, sino la combinación de varias capacidades en estructuras adaptadas a una situación particular. Este concepto remite para la existencia de una interacción dinámica entre los elementos constitutivos de la competencia, bien como articulación entre estos y la acción, a través de la cual la competencia se expresa. 
Kirschner et al. (1997) entienden que ser competente exige conocimiento y habilidad, pero no se confina a comportamiento previsibles en situaciones previsibles. Los autores están de acuerdo que tratar con lo inesperado y lo imprevisible de una manera creativa es un componente crítico del concepto de competencia. La competencia envuelve estrategia, o sea, la capacidad de elegir, combinar o reconfigurar un conocimiento y habilidades en función de una evaluación y conocimiento de la situación. En una fórmula sintética, la competencia $(\mathrm{C})$ puede concebirse como función ( $\mathrm{f}$ ) del conocimiento $(\mathrm{K})$, de las habilidades (Sk) y de la situación $(\mathrm{S})$, esto es: $=\mathrm{f}(\mathrm{K}, \mathrm{Sk}, \mathrm{S})$. Kirschner et al.(1997) definen competencia como: "la capacidad de tomar decisiones satisfactorias y eficaces en una situación específica" (p. 155) ${ }^{[6]}$. En la perspectiva de los autores, además de revelarse en la actuación eficaz y apropiada en circunstancias marcadas por la imprevisibilidad y por el cambio, la competencia presupone juicio, valores y autoconfianza para asumir riesgos y voluntad de aprender con la experiencia.

En el ámbito de la preparación profesional, Mansfield (2004) dice que la persistencia de una visión estrecha de competencia está basada en una idea sobrepasada de eficiencia y eficacia, reportando a la aplicación de reglas y procedimientos circunscritos a tareas rutinarias, típicas de los procesos industriales de producción en masa. A este entendimiento estrecho, el autor contrapone una visión más amplia de competencia profesional en la cual las personas son llamadas a asumir mayor responsabilidad para responder a exigencias de responsabilidad, adaptabilidad, multifuncionalidad, polivalencia, versatilidad requeridas por los cambios en la economía.

Aoki (1984), posicionándose en una perspectiva social crítica, contrapone a una competencia entendida como acción instrumental (es decir, acción de seres como cosas, orientada en función del interés en el control, la eficiencia y la certeza) una concepción radicalmente distinta de competencia, una competencia entendida como acción práctica, como praxis (es decir, acción de seres-como-humanos, orientada en función del interés en la comprensión mutua y preservación de la autenticidad de la ínter subjetividad).

En la perspectiva de acción instrumental, la realidad se objetiva, esto es, se entiende como algo que está regido por leyes, que justifican los padrones acción racional orientada por propósitos de eficiencia y eficacia, de competencia técnica confinada a la relación medio-fin, sin cuestionar la razón de los fines.

En la perspectiva de acción práctica, la realidad está constituida por una comunidad de actores y habladores, que actúan y reflejan sobre la acción, haciendo un esfuerzo consciente para examinar las intenciones y asunciones que están subyacentes a las acciones y buscar, en conjunto, alternativas de emancipación y mejora de la vida personal y social.

Carr (1993) sustenta que el concepto de competencia engloba dos componentes: un componente teleológico, en el sentido aristotélico de causa final, y un componente causal, en el sentido de causa eficiente. La gran dificultad conceptual, reside en la forma de conciliar satisfactoriamente estos dos componentes. El sentido teleológico remite para la dimensión normativa de competencia, para una comprensión cualitativa de objetivos racionales y propósitos, para el ejercicio de la capacidad de

[6] "the ability to make satisfactory and effective decisions in a specific setting or situation". 
juzgar y de actuar de forma libre, voluntaria y deliberada a la luz del conocimiento racional y la comprensión, para padrones, cánones y criterios de evaluación, de realización y de éxito. El sentido del poder causal, por su lado, remite para una dimensión de eficacia causal y eficiencia práctica, para una operatividad, para poderes naturales o adquiridos por el aprendizaje, experiencia y entrenamiento, para un "saber cómo", para facultades, habilidades, procedimientos, técnicas, hábitos y rutinas que nos permiten realizar con eficacia y eficiencia determinadas funciones.

Si para Carr (1993) "la competencia profesional debe evaluarse tanto en términos de comprensión y discernimiento del profesor acerca de lo que está haciendo, como en términos de pura eficacia causal de aquello que hace" (p. 270), este autor no deja de considerar éticamente objetable, políticamente indeseable y conceptualmente incoherente negar la importancia de la componente teleológica de la formación a favor de una incidencia en la adquisición y entrenamiento de competencias técnicas, y en esta medida, reduciendo toda y cualquier tentativa de concebir la construcción de competencia profesional con base en la adquisición y entrenamiento de competencias genéricas o de habilidades especificas inventariables.

Tarrant (2000) Ilega a una conclusión en todo semejante, cuando cuestiona lo que está mal en el concepto de competencia y afirma que "toda cuestión de exigencia de una preparación para el trabajo a costa de la ciudadanía, del desarrollo conceptual y de una concepción más amplia de persona que la de empleado es completamente controvertida y éticamente inaceptable" (p.83)

Hyland $(1993 ; 1997)$ va mas allá cuando rechaza la posibilidad de reconvertirse el concepto de competencia en algo ventajoso para la educación y formación. En las palabras del autor la competencia es un eslogan ideal para una visión tecnócrata (managerialist and technicist), en la medida en que se hace combinar un sistema de evaluación aparentemente objetivo y preciso con los requisitos de control y prestación de cuentas.

Por otro lado, Holmes $(1992 ; 2000 ; 2001)$ está convencido de la utilidad del concepto de competencia en el ámbito de educación y de formación profesional, pero reclama, sin embargo, la necesidad de proceder al rescate del concepto a través de una clarificación que denote los elementos de confusión y la contaminación de su significado cuando se cambia de contexto discursivo. Según el autor, además de los diferentes contextos de utilización, los discursos acerca de la competencia son, también estos, diferentes en el modo y en el propósito pertenecen a distintos "juegos de lenguaje". Así, el discurso descriptivo es factual, da cuenta de cómo son las cosas; el discurso explicativo es causal, da cuenta de las relaciones empíricas entre los acontecimientos y sus antecedentes; y el discurso evaluativo es de orden de lo normativo, y da cuenta de la distancia, relativamente a lo deseado, engloba el juicio de valor a cerca de algo o de alguien. Reconocer la competencia de alguien es el resultado de una inferencia sobre la actuación futura con base en la información recogida hasta el momento:

[7] The whole matter of demanding a preparation for work at the expense of citizenship, conceptual development, and a wider conception of the person than that of an employee, is entirely controversial, and arguably, unethical. 
Nosotros atribuimos competencia a alguien en una dada área o profesión, cuando inferimos a partir de la información que tenemos normalmente, pero no exclusivamente basada en el desempeño pasado o actual, que situará el desempeño futuro a un nivel del deseado o requerido. Hacemos esa inferencia con base en los datos que poseemos aplicando reglas de inferencia implicitas o explicitas. Tales reglas pueden contar cómo la información recogida puede utilizarse; pueden contar también qué tipo de información puede recogerse. Con todo, no existen cualesquiera enunciados objetivos de lo que constituye el desempeño deseado o requerido, existen sólo enunciados de aquellos que reclaman el derecho de prescribir el desempeño y de producir inferencias de competencia apropiadas. (Len Holmes, 1992, p.7 (8]. $^{[8]}$

Holmes (1992) rechaza así que la competencia sea una entidad discreta que se posea; una característica inmediatamente observable o cualquier herramienta tipo ordenador lista a usar.

Short (1984) analizó el concepto de competencia asumiendo como punto de partida su naturaleza normativa y su dependencia del significado de la actividad de referencia o contexto de aplicación. Aunque se centra en las cuestiones relacionadas con la enseñanza, tuvo la preocupación de presentar referencias que pueden generalizarse. De acuerdo con el análisis que realizó, Short llegó a cuatro concepciones normativas de competencia, que pueden traducirse en las formas siguientes:

Competencia bajo la forma de comportamientos o desempeños, es decir, estar apto a demostrar competencia en un comportamiento o desempeño particular es, necesariamente, estar apto para demostrar algo muy específico y limitado. La utilidad y la aplicación de este entendimiento de competencia son de ámbito muy restringido.

Competencia bajo la forma de conocimientos y habilidades: esta forma implica no sólo comportamiento o desempeño, sino también ser capaz de escoger y justificar el porqué de la elección. La selección del conocimiento y las habilidades y la determinación del momento de su utilización en una situación específica, se guían por el propósito o intención que la persona tiene en esa situación específica. La concepción de competencia, aunque es más amplia que la anterior, no permite llegar a una "declaración" de competencia. La ausencia de "cantidad" de lo que se considera suficiente para decir/asumir que "alguien" es competente, es el gran fallo.

Competencia bajo la forma de juicio sobre el grado o nivel de capacidad, considerada como suficiente, en una determinada categoría o actividad particular. Esta concepción es más comprensiva si la comparamos con las anteriores, incorpora el léxico de valores, evaluación, que es una dimensión inherente a la noción de competencia y se puede aplicar. Las entidades se juzgan teniendo en cuenta comportamientos, conocimientos, objetivos y resultados u otros aspectos.

[8] We attribute competence to someone in a particular area or occupation when we infer from information we have, usually but not exclusively based on past or current performance, that future performance will accord with desired or required performance. We make such an inference on the basis of the evidence we have using implicit or explicit rules of inference. Such rules may indicate how the information from the evidence may be used; they may also indicate what type of information should be obtained. However there are no objective statements of what constitutes desired or required performance, only statements of those who claim the right to prescribe performance and to make appropriate inferences of competence. 
Competencia bajo la forma de cualidades de la persona o estados de ser. En esta concepción, es necesario comprender esas cualidades de manera holística, lo que implica plantear las interrelaciones de todas las dimensiones de la actividad, incluso conductas, desempeños, conocimientos, habilidades, niveles de suficiencia, además de intenciones, motivos, o actitudes en referencia a una concepción de competencia como un todo. Una vez determinada la naturaleza de tales cualidades seremos capaces de evaluar si una persona las posee o no.

El autor asume esta concepción de competencia basada en las cualidades como la más susceptible de aplicación y generalización.

En los estudios que realizó en el ámbito de evaluación educacional, Weinert (1999) deparó una diversidad interminable de usos científicos del concepto de competencia y una gran variedad de aproximaciones teóricas, pero ningún marco conceptual común. Weinert llegó a una lista descriptiva que apunta para nueve aproximaciones distintas ${ }^{[9]}$, cada cual con sus formas propias de definir e interpretar la competencia. A fin de aprehender las diferencias de las varias teorías y perspectivas del concepto de competencia, el autor postula la discriminación de al menos siete usos distintos de competencia. Éstos pueden sintetizarse de la siguiente forma:

competencia como capacidad cognitiva general (modelos psicométricos de inteligencia (C.I.), modelos de procesamiento de información, estadios de desarroIlo cognitivo) - se refiere a recursos cognitivos que se aplican a un conjunto de tareas transversales o a distintos contenidos.

competencia como capacidad cognitiva especializada - se asume que para el desempeño superior en una determinada área, los recursos cognitivos (competencias) son de naturaleza específica en vez de general.

competencia en términos de motivación - contructos tales como autoconcepto, autoeficacia y demás creencias y evaluaciones subjetivas de sí mismo, de sus recursos personales, sean generales o específicas, sean estables o instables, condicionan la motivación para la acción, y consecuentemente el desempeño de los sujetos.

competencia de acción - considera al mismo tiempo las vertientes objetivas e subjetivas de la competencia, o sea considera los recursos de naturaleza cognitiva y motivacional requeridos para encarar los objetivos, las exigencias y las tareas dentro del contexto particular de ión.

competencias clave-son competencias transversales que conllevan a buenos desempeños a lo largo de un amplio espectro de situaciones, y por lo tanto deben estar incluidas en el proceso educativo básico (literacia, numeracia, competencias comunicativas, competencias tecnológicas, competencias de planeamiento, toma de decisión y resolución de problemas).

metacompetencias - competencias de inspeccionar y regular los propios procesos cognitivos, de reflexionar sobre los propios procesos de aprendizaje y valorar las estrategias más convenientes.

[9] General cognitive ability; specialized cognitive skill; competence-performance model; modified competence-perfomance model; objective and subjective self-concepts; motivated action tendencies; action competence, key competencies; meta-competencies. 
competencias individuales son solo una pequeña parte de los recursos humanos totales necesarios al desarrollo de una sociedad, economía o institución.

A pesar de la diversidad de entendimientos, usos y contextos de aplicación, Harzallah et al. (2002), en un esfuerzo de síntesis, consiguieron identificar puntos de convergencia en la literatura que, de alguna manera, muestran lo que hay de fundamental en el concepto de competencia:

Se distinguen usualmente dos tipos de competencia: la competencia requerida por el sistema y la competencia adquirida (o suministrada) por cada persona perteneciente al sistema.

La competencia es constituida por recursos estructurados en categorías y subcategorías. De acuerdo con la literatura, éstas consideran tres categorías fundamentales: conocimiento, saber hacer (know-how) y comportamientos, que a su vez se pueden subdividir en diversas subcategorías.

La competencia siempre se relaciona con un contexto dado, que concierne la situación en la cual la competencia se experimenta y que incluye los aspectos físicos y los aspectos inmateriales del sistema estudiado.

La competencia está relacionada con la realización de una o más misiones o tareas. Estas misiones o tareas constituyen la finalidad de la competencia.

Además de estas características referenciadas por los autores, podemos todavía apuntar otras características relevantes:

el uso de la competencia surge íntimamente asociado a la actividad profesional, pareciendo ser éste el elemento unificador.

la naturaleza del concepto es relacional, pues incorpora atributos y tareas y resulta de la relación entre las capacidades y potencialidades de personas y la conclusión satisfactoria de una determinada tarea.

conocimiento y habilidades son atributos presentes en la generalidad de varios conceptos de competencia; el conocimiento tiende a ocupar un lugar de relieve.

La actitud surge como atributo de la competencia, pero con estatutos diferentes de acuerdo con la conceptualización de competencia: o se asume como una categoría o extrapola la "composición" de la competencia, asumiéndose como elemento determinante de la competencia.

La competencia no es algo directamente observable, sólo puede inferirse a partir de la interpretación de desempeños.

La competencia posee un vínculo de orden normativo, en la medida en que implica siempre un juicio de valor a cerca de lo que es deseable y apropiado. 


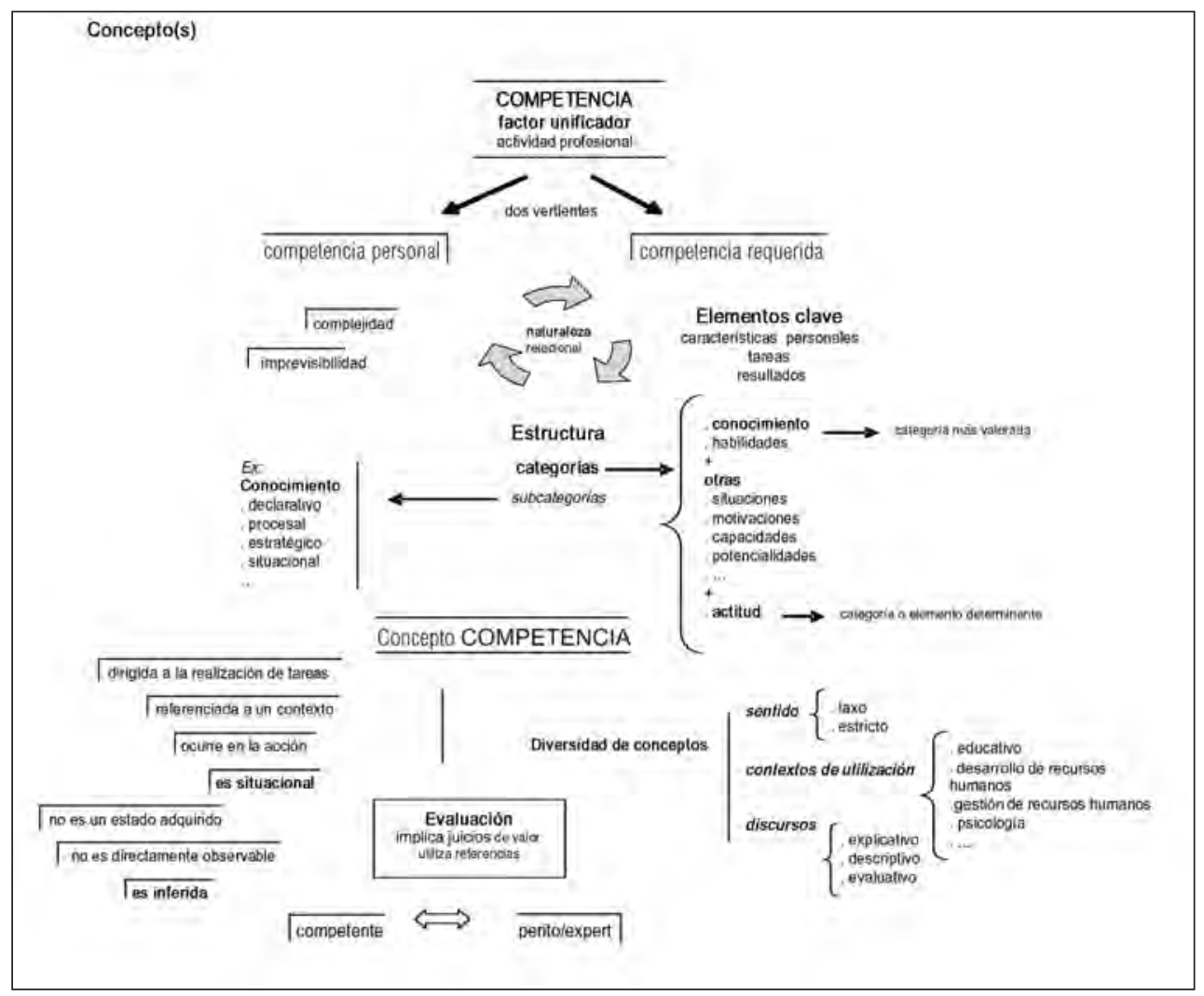

Figura 2. Síntesis de los aspectos relacionados con el concepto de competencia.

Recuperando el rescate de la idea de Burgoyne (1976) a propósito de competencia, constatamos que ésta, en lo esencial, permanece muy actual. El autor considera que la competencia no es una entidad, una idea, o cualidad percibida, sino un concepto que tiene en cuenta la condición o modo de ser, cuando miramos las circunstancias, la relación (circunstancias concretas en que las relaciones se establecen). La competencia surge así como un concepto complejo, relacional, holístico e integrativo. El hecho de que un individuo posee los "compuestos" que integran la competencia, no es garantía de ser competente. La competencia es situacional, ocurre en la acción, no siendo directamente observable y, aunque se relaciona con el éxito, difiere mucho de "eficiencia", de desempeño. La competencia no es un estado adquirido, se relaciona con un contexto determinado, no puede utilizarse como indicador de competencia futura. La competencia sólo puede inferirse por la interpretación de múltiples desempeños que tengan en cuenta su naturaleza situacional, es decir, su íntima relación con la acción (realización de tarea(s), misión(es), en un determinado contexto).

Los datos presentados nos permiten clarificar el concepto de competencia. El significado de competencia, se relaciona íntimamente con el desempeño eficaz y con 
la persona que lo desempeña, el concepto de competencia se aleja del desempeño eficaz puntual, dado que aunque se manifiesta en la acción concreta, es situacional por lo que no existe garantía de que se repita. La competencia, siendo una manifestación del sujeto, sufre influencia no sólo de variables intrínsecas sino también de variables extrínsecas, sobre todo el contexto donde se manifiesta. Hay que referir además que, siendo la dimensión individual una característica del concepto de competencia, la dimensión colectiva no deja de estar presente. Utilizando como ejemplo la actividad profesional, diremos que el individuo tiene que ser capaz de interpretar la organización (su misión, valores, estructuración,...) de forma a interiorizar el colectivo en el que está integrado para que, de esta forma, la competencia pueda manifestarse. Corroborando el entendimiento de autores como Stoof, Martens, Van Merriënboer, \& Bastiaens (2002) la competencia, como constructo teórico, no existe, pero este hecho no le quita importancia, en la medida en que nos deparamos constantemente con cuestiones relativas a la competencia. Esta circunstancia remite para la necesidad de movilizar más esfuerzos en la realización de trabajos de naturaleza teórica y no para soluciones apresuradas, sobre todo la no utilización o sustitución del término competencia por el término competencias, como sugiere Parente (2003). Independientemente de la utilidad del término competencias, este es distinto de competencia. Seguimos la idea de Burgoyone (1988) que defiende que "ser competente" es muy diferente a "tener competencias". Los dos términos son útiles, pero no pueden sobreponerse y, por supuesto, tampoco sustituirse.

\section{Conclusiones}

Al repasar el contenido del trabajo, importa sintetizar los siguientes aspectos:

- no existe un solo constructo teórico de "competencia", coexisten varios en campos conceptuales diferenciados;

- la competencia es un término usado de forma indiferenciada, con distintos significados y en múltiples contextos;

- podemos distinguir significado de concepto. El significado se relaciona con las cuestiones lingüísticas y está directamente relacionado a la palabra y el concepto se relaciona con el término y se refiere a un ámbito específico de la realidad;

- la palabra "competencia" tiene como foco a la persona, asume diferentes significados, se reporta a una actividad concreta realizada con éxito, surgiendo en estrecha asociación con la actividad profesional;

- el concepto de competencia es un concepto complejo, relacional, holístico e integrativo. La competencia es situacional, ocurre en la acción, no siendo directamente observable. Se relaciona con el éxito pero no se confunde con el desempeño concreto;

- en la generalidad de las definiciones de competencia los atributos "conocimiento" y "destrezas", están presentes. En otras definiciones, más explicativas, aparecen también atributos como capacidades, motivaciones y actitudes, entre otros;

- aunque el estudio de la competencia se remonta a los años 20 del siglo pasado, las cuestiones en torno al concepto de competencia necesitan todavía mucha clarificación. 


\section{Aportes para el futuro}

Consideramos que la investigación, en el área de la competencia, debe asumir nuevos rumos y nuevas formas de considerar teóricamente su debate, asumiendo nuevos paradigmas que respondan a las necesidades actuales que se colocan en su estudio. La búsqueda de una definición de competencia consensual puede revelarse inútil.

La ausencia de unanimidad teórica, no impide el estudio de la competencia, pero obliga a situar el paradigma adoptado, cualquier que sea el aspecto de la competencia a investigar. Importa desarrollar más y mejores esfuerzos en la clarificación del campo nocional ${ }^{[10]}$, y en la descontaminación conceptual de los atributos presentes en la competencia. Tal y como el concepto de competencia, también los atributos, que componen la competencia, como conocimiento, destrezas, actitudes, entre otros, sufren contaminación conceptual. Otro aspecto importante es investir en las cuestiones relacionadas con la adquisición y desarrollo de la competencia en busca de indicaciones para la elaboración de programas de formación inicial y desarrollo profesional.

Tomando como referencia los aspectos fundamentales de la "competencia" expuestos, podemos afirmar que es imprescindible que los procesos de formación y desarrollo de la competencia permitan la aprehensión de la dimensión situacional de la competencia. El sujeto en formación tiene que vivir y comprender este proceso. Es imprescindible, en la formación inicial, trabajar la reflexión, la capacidad de decisión, la resolución de problemas, la creatividad, la agilidad mental... porque son herramientas fundamentales para que el futuro profesional sea capaz de dar respuesta a las exigencias de la sociedad y de las futuras situaciones de trabajo No olvidemos que, hoy en día, los conocimientos son cada vez más complejos y pierden rápidamente actualidad, tornándose casi imposible aprehender toda la información, evaluarla, procesarla y retenerla. Claramente, los procesos de educación y formación, tienen que incorporar la complejidad y la temporalidad, utilizando metodologías que conduzcan a comportamientos intencionales y conscientes.

Es en este contexto que los modelos tradicionales de formación, basados en el entrenamiento y reproducción de acciones demostrativas de la adquisición de determinadas competencias, pierden sentido. Lo que está en causa no es sólo la función pero también la propia persona, sus objetivos y posibilidades en el proceso de construcción de su competencia. Los escolares tienen que envolverse en su proceso de formación y ganar autonomía en la construcción de su competencia, para poder desarrollarla sobre todo a través de la formación continua que debe ser frecuente y cuidadosamente elegida.

[10] campo nocional de una doctrina - conjunto de terminología preexistente a la doctrina, cualquier que fuese el dominio al que perteneciese previamente, pudiendo tratarse de palabras del idioma o de expresiones medio conceptualizadas. Es necesario verificar siempre cual fue la significación anterior de la noción, el campo al que perteneció, a fin de medir la desviación provocada por su integración en nuevo contexto. 


\section{Bibliografía}

Aoki, T. (1984). Competence in teaching as instrumental and practical action: a critical analysis. En E. C. Short (Ed.), Competence inquires into its meaning and aquisition in educational settings (pp. 71-82). Lanham: University Press of America.

Boak, G. (1991). Developing Managerial competences: The management learning contract approach. London: Pitman.

Burgoyne, J. (1976). Managerial Effectiveness Revisited. University of Lancaster.

Burgoyone, J. (1988). Competency Based Approaches to Management Development. Lancaster: Centre for the Study of Management Learning.

Cabré, M. (2000). Terminologie et linguistique: la théorie des portes. Terminologies Nouvelles, 21, 10-15.

Carr, D. (1993). Questions of competence. British Journal of Educational Studies, 41(3), 253-271.

De Landsheere, G. (1986). A Investigação Experimental em Pedagogia. Lisboa: Publicações D. Quixote.

Ecke, P. (1981). Undersuchungen Zum Pädagogischen Können. Berlin: Yolk und Wissen Volkseigener Verlag.

Flach, H. (Ed.) (1986). Zur Entwicklung des Pädagogischen Könnens in der Lehrerausbildung Berlin: Volk und Wissen Volkseigener Verlag.

Flew, A. (Ed.) (1979). A Dictionary of Philosophy. London: Macmillan.

Gaudin, F. (1995). Champs, clôtures et domaines; des langues de Spécialités à la culture scientifique. Meta, 40(2), 224-228.

Grossman, P. (Ed.). (1990). The Making of a Teacher. Teacher and teacher Education. New York: Teachers College Press.

Hager, P. (1993). Conceptions of competence [Electronic Version]. Philosophy of Education Yearbook 1993. Retrieved 2001, 21 Feb from T COMP.

http://www.ed.uiuc.edu/eps/pes-yearbook/93_docs/hager.htm

Hager, P. \& Gonczi, A. (1996). What is competence? Medical Teacher, 18(1), 15.

Harzallah, M., Berio, G., \& Vernadat, F. (2002). A formal model for assessing individual competence in enterprises. Paper presented at the 2002 IEEE International Conference on Systems, Man and Cybernetics.

Hoffmann, T. (1999). The meanings of competency. Journal of European Industrial Training, 23(6/7), 275-285.

Holmes, L. (1992). Understanding professional competence: Beyond the limits of functional analysis. Paper prepared for Course Tutors' Conference, Institute of Personnel Management at UMIST, 6-8 July 1992 [Electronic Version].

Holmes, L. (2000). What can performance tell us about learning? Explicating a troubled concept. European Journal of Work and Organizational Psychology, 9(2), 253-266.

Holmes, L. (2001). Decontaminating the concepts of 'learning'and 'competence': education and modalities of emergent identity. Paper presented at the Second International Conference on Critical Management Studies, Manchester.

Hyland, T. (1993). Professional development and competence-based education. Educational Studies (03055698), 19(1), 123. 
Hyland, T. (1997). Reconsidering Competence. Journal of Philosophy of Education, 31(3), 491-503.

Kirshhner, P., Vilsteren, P.; Hummel, H., \& Wigman, M. (1997). The design of a study environment and professional competence. Studies in Higher Education, 22(2), 151171.

Lara, M. (2004). Diferenças conceptuais sobre termos e definições e implicações na organização da linguagem documentária. Ciência da Informação, 33(2), 91-96.

Le Deist, F., \& Winterton, J. (2005). What Is Competence? Human Resource Development International, 8(1), 27-46.

Mansfield, B. (2004). Competence in transition. Emerald Journal of European Industrial Training, 28(2/3/4), 296-309.

Matos, Z. (1989). Para uma definição do conceito e dos pressupostos do desenvolvimento da competência pedagógica. Porto: Universidade do Porto.

Noddings, N. (1984). Competence in teaching: a linguistic analisys. En E. C. Short (Ed.), Competence inquires into its meaning and aquisition in educational settings (pp. 1728). Lanham: University Press of America.

Parente, C. (2003). Construção social das competências profissionais: dois estudos de caso em empresas multinacionais do sector metalomecânico. Porto: Faculdade de Letras.

Parry, S. (1996). The quest for competencies. Trainning, 48-56.

Pearson, A. (1984). Competence: A normative analysis. En Competence inquires into its meaning and aquisition in educational settings. (pp. 31-40). Lanham: University Press of America.

Perrenoud, P. (1999). Pedagogia Diferenciada. Das Intenções à Ação. (trad. en portugués de Pédagogie différenciée : des intentions à I'action. Paris: ESF, Trans.). Porto Alegre: Artmed Editora.

Porto Editora (Ed.) (2007). Dicionário de Lingua Portuguesa. Porto: Porto Editora.

Short, E. (1984). Competence Reexamined. Educational Theory, 34(3), 201-207.

Shulman, L. (1987). Assessment for teaching: an initiative for the profession. Phi Delta Kappan, 69(1), 38-44.

Spencer, L.; Spencer, M. (Ed.) (1993). Competence at Work: Models for superior performance. United States of America: John Wiley \& Sons, Inc.

Stoof, A., Martens, R. L., van Merriënboer, J. J., \& Bastiaens, T. J. (2002). The Boundary Approach of Competence: A Constructivist Aid for Understanding and Using the Concept of Competence. Human Resource Development Review, 1(3), 345-365.

Tarrant, J. (2000). What is Wrong with Competence? Journal of Further and Higher Education, 24(1), 77-83.

Tate, W. (1995). Developing Managerial Competence: A critical guide to Methods and materials. London: Gower.

Weinert, F. (1999). Definitions and Selection of Competencies. Concepts of competence. Munich: Max Planck Institute for Psychological Research.

Winterton, J., Winterton, R. (Ed.) (1999). Developing Managerial Competence. London: Routledge.

Woodruffe, C. (1991). Competence by any other name. Personal Management, 30-33. 